\title{
Scrutiny of injected electron current polarization effect on the threshold current density reduction in QW spin-lasers
}

\author{
S. N. Hosseinimotlagh ${ }^{1}$, H. Ghavidelfard ${ }^{2}$, M. Pezeshkian ${ }^{3}$, H. Molaei $^{3}$ \\ ${ }^{1}$ Department of Physics, Shiraz branch Islamic Azad University, Shiraz, Iran \\ ${ }^{2}$ Department of Physics, Payam Noor University, Bandar Abbas, Iran \\ ${ }^{3}$ Department of Physics, Payam Noor University, Shiraz, Iran
}

\section{Email address:}

hoseinimotlagh@hotmail.com (S. N. Hosseinimotlagh)

\section{To cite this article:}

S. N. Hosseinimotlagh, H. Ghavidelfard, M. Pezeshkian, H. Molaei. Scrutiny of Injected Electron Current Polarization Effect on the Threshold Current Density Reduction in QW Spin-Lasers. American Journal of Electromagnetics and Applications.

Vol. 2, No. 2, 2014, pp. 16-22. doi: 10.11648/j.ajea.20140202.12

\begin{abstract}
One of the mechanisms for threshold current density reduction is using spin polarized carriers generated by electrical spin injection. Electrical spin injection is spin-polarized carrier injection by using a magnetic contact. In this paper, we have solved numerically rate equations governing on semiconductor spin un-polarized and polarized laser with $I n_{0.2} G a_{0.8} A s$ - based quantum well active region in which $\mathrm{Fe} / A l_{0.1} G a_{0.9} A s$ Schottky tunnel barrier treat as the spin injector. For the first time, we demonstrate simultaneously effect of normalized spin relaxation rate and injected current polarization on threshold current density reduction related to two form of spontaneous recombination. According to our result threshold current density reduction increases by simultaneously normalized spin relaxation rate reduction and increasing of injected current polarization. Maximum obtained threshold current density reduction values for linear and quadratic spontaneous recombination is 0.07 and 0.31 . Moreover, we compute and compare the effect of value of injected electron current polarization on normalized spin-filtering interval for two types of recombination. Maximum obtained normalized spinfiltering interval values for linear and quadratic spontaneous recombination is 1.2 and 1.36. Finally we calculate spin-up optical gain and from this we obtained the conditions for achieving optimum optical gain. Maximum obtained spin-up optical gain valueis17.36.
\end{abstract}

Keywords: Spin Laser, Gain, Threshold Current, Quantum Well, Filtering

\section{Introduction}

The importance of lasers generally reflects to their practical applications [1-5]. Semiconductor lasers are important due to its widespread applications. Semiconductor lasers use semiconductor as active medium. An active material is pumped to create population inversion and light can be amplified through stimulated emission. By introduction of spin-polarized carriers which is physical mechanisms that enhance stimulated emission, we can reduce current density threshold in semiconductor lasers [613].Such semiconductor lasers are called semiconductor spin polarized-lasers (SSPLs) [14-16].

Most of the SSPLs are vertical cavity surface emitting lasers (VCSELs) with active region consisting of III-V Quantum wells (QWs).The VCSELs are type of semiconductor lasers with laser beam emission perpendicular. A VCSEL should have a resonant cavity with two distributed Bragg reflectors (DBRs).A DBR is light reflecting device based on Bragg reflections in a periodic structure, alternating high and low refractive indices and with quarter-wave length material thick. It must be highly optically reflective and electrically conductive. Advantages of such laser are low power consumption, low threshold currents and generate less heat, but it also have lower output power than other semiconductor lasers. In this paper, we intend to investigate simultaneously effects of injected current polarization and normalized spin relaxation rate on threshold current density reduction (TCDR)in a QW-SSPL by its numerical rate equation solution. For achieving this aim, we introduce spin polarized injection and present proper materials for implement that (section 2). In section 3 have been represented numerically solution of rate equations governing on $\operatorname{In}_{0.2} G a_{0.8} A s$ QWs based spin polarized and un-polarized semiconductor laser. As creation 
of normalized spin-filtering interval (NSFI) and TCDR are two important consequences of spin polarized injection, we compute the effects of injected electron current polarization on NSFI in section 4. Section 5 shows simultaneously the effects of injected electron current polarization and normalized spin relaxation rate on TCDR. In section 6 , we calculate spin-up optical gain and from this we obtain the conditions for achieving optimum optical gain. Finally, we present conclusion and discussion in section 7.

\section{Suitable Materials for Spin-Polarized Injection}

Spin-polarized electron injection into semiconductors has been a field of growing interest during the last years [17].As we need electron injection for electronic devices, spintronics devices require spin-polarized electron injection. A spin-polarization of the current is expected from the different conductivities resulting from the different densities of states for spin-up and spin-down electrons in the ferromagnetic materials. Comfortable way to create spin-polarized electron injection is passing electron current form ferromagnetic materials. Spin injectors are materials which create spin-polarized electron injection. There have been many choices for spin injectors but the most obvious choice are ferromagnetic materials due to their high Curie temperatures, low coercivities and fast switching times. The main problem for using ferromagnetic materials is conductivity mismatch which occurs at the interface between ferromagnetic and semiconductor materials [18].There are three solutions to solve this problem [19]. The first solution is to use a half metallic ferromagnets [20] which are materials that possess a bandgap at the Fermi level for one of the spin sub-bands, generally the minority-spin sub-band, making them $100 \%$ spin-polarized. The second solution is to use dilute magnetic semiconductors which have similar conductivity with magnetic materials. Note that Curie temperature of these materials is still well below room temperature .Another solution is to use either an extrinsic or intrinsic tunnel barrier. The advantage of using a tunnel barrier is that it allows ferromagnetic materials to be used as the source of spin-polarized electrons. An intrinsic Schottky barrier is formed when a ferromagnetic material is placed in contact with a semiconductor. It overcomes limitations of the conductivity mismatch without the need for the deposition of a tunnel barrier.

\section{Rate Equations}

The electronic transitions take place between conduction and valence band carriers in QW-SSPLs. The first of the processes is spontaneous recombination of an electron in the conduction band and a hole in the valence band which results in incoherent emission. The second process is the photon absorption by the active material which promotes the generation of an electron-hole pair and increases the carrier density in both the conduction band and valence band. The third transition is the emission of a photon by means of an electron-hole recombination after the stimulus of another photon already present in the cavity. This process provides optical gain because it starts with one photon and ends with two photons. The dynamics of carrier and photon densities in semiconductor lasers are governed by the coupled rate equations. Rate equations describe how electrons and holes turn into photons.

For simplicity, let us assuming a constant current injection rate i.e. at each unit time amount of electron is injected into the laser active region. This pumping process increases the number of electrons in the conduction band and holes in the valence band. Photon absorption in the semiconductor material increases the number of electrons in the conduction band and holes in the valence band. The number of electrons in the conduction band and holes in the valence band is reduced by photon-emitting processes. The photon-emitting processes are those which generate photons through spontaneous recombination and stimulated emission. Stimulated emitted photons and the spontaneous recombination processes will contribute to increase the photon density, because these processes are producing light inside the device, whereas the photons involved in the stimulated absorption processes cause he opposite effect, thus decreasing the photon number in a time interval. Besides the stimulated absorption, also the material optical loss will reduce the photon density and expresses how many photons are lost as they propagate at each centimeter of the cavity and as they impinge on the cavity end mirrors. By assumption of neutrality of charge and decoupling rate equation for hole densities, rate equation of $\mathrm{QW}$ semiconductor laser in terms of electron $(n)$ and photon $(S)$ densities is written as $[21,22]$

$$
\begin{gathered}
\frac{d n}{d t}=J-g_{0} \frac{\left(n-n_{\text {tran }}\right)}{1+\varepsilon S} S-R_{s p}(n) \\
\frac{d S}{d t}=\Gamma g_{0} \frac{\left(n-n_{\text {tran }}\right)}{1+\varepsilon S} S-\Gamma \beta R_{s p}(n)-\frac{S}{\tau_{p h}}
\end{gathered}
$$

Where $J, g_{0}, n_{\text {tran }}, \mathcal{E}, R_{s p}(n), \Gamma, \tau_{p h}$ and $\beta$ are carrier injection rate, gain coefficient, transparency density, gain compression factor, recombination function, optical confinement factor, photon lifetime and spontaneous emission factor, respectively. We investigate linear (LR) and quadratic $(\mathrm{QR})$ form of spontaneous recombination with mathematical forms as $R_{s p}(n)=p n B / 2$ and $R_{s p}(n)=n / \tau_{r}$ where $B$ and $\tau_{r}$ is a temperature dependent constant and recombination time, respectively. Note that $g_{0} \frac{\left(n-n_{\text {tran }}\right)}{1+\varepsilon S}$ represents optical gain term. Fig. 1 shows time dependency of electron densities by considering LR spontaneous recombination. 


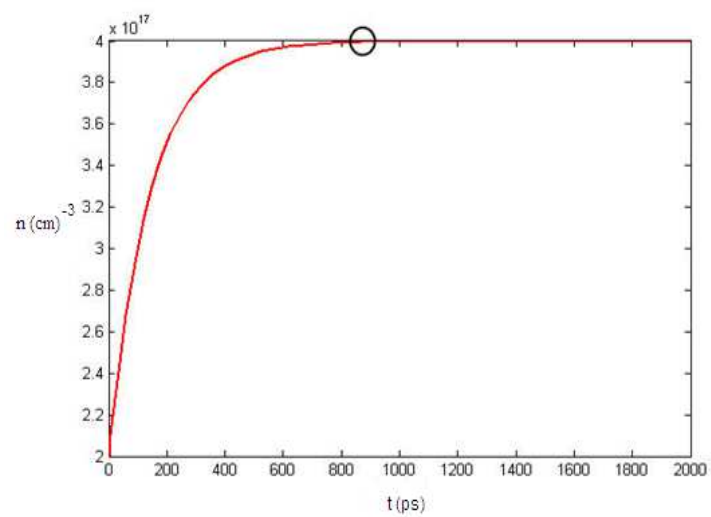

Fig 1. Time dependency of electron densities in a $Q W$ semiconductor laser.

According to this figure, we find out increase of electron densities at constant spin un-polarized electron injection $10^{15}$ per every second by considering LR spontaneous recombination .As shown in Fig. 1,from point $(870.0558$, $\left.4 \times 10^{17}\right)$ which is characterized with black circle, electron density have constant trend and saturation reaches. Table 1 illustrates obtained values of electron densities at various times.

Table 1. Obtained values of electron densities at various times

\begin{tabular}{cccc}
\hline $\mathbf{n}\left(\mathbf{c m}^{-3}\right)$ & $\mathbf{t}(\mathbf{p s})$ & $\mathbf{n}\left(\mathbf{c m}^{-3}\right)$ & $\mathbf{t}(\mathbf{p s})$ \\
\hline $2.68 \times 10^{17}$ & 59.0479 & $3.87 \times 10^{17}$ & 395.4563 \\
$3.30 \times 10^{17}$ & 150.0699 & $3.97 \times 10^{17}$ & 620.0558 \\
$3.55 \times 10^{17}$ & 214.4130 & $4 \times 10^{17}$ & 870.0558 \\
\hline
\end{tabular}

Fig 2. demonstrates time dependency of photon densities by considering LR spontaneous recombination.

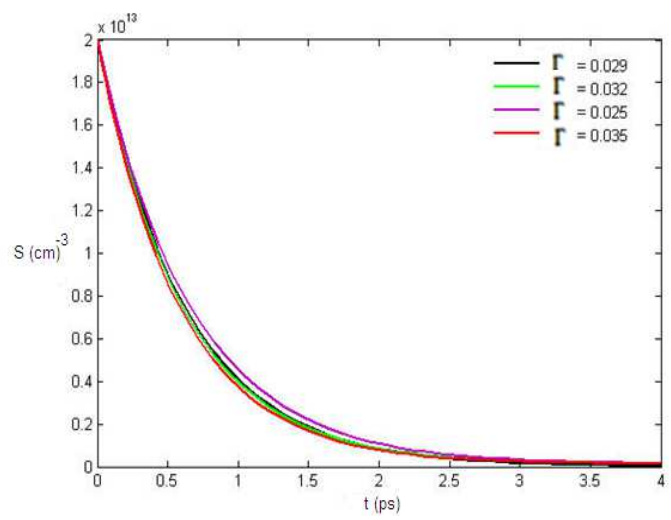

Fig 2. Time dependency of photon densities in $Q W$ semiconductor laser.

According to above figure, we find out decrease of photon densities at constant spin un-polarized electron injection $10^{15}$ per every second by considering LR spontaneous recombination. As shown in Fig. 2, we note that by increase of optical confinement factor, drop of photon densities occur slower. Table 2 illustrates obtained values of photon densities for three various optical confinement factors at various times.
Table 2. Obtained values of photon densities for three various optical confinement factors at various times

\begin{tabular}{cccccc}
\hline \multicolumn{2}{c}{$\boldsymbol{\Gamma = 0 . 0 2 9}$} & \multicolumn{2}{c}{$\boldsymbol{\Gamma}=\mathbf{0 . 0 3 2}$} & \multicolumn{2}{c}{$\boldsymbol{\Gamma = 0 . 0 3 5}$} \\
$\mathbf{S}\left(\mathbf{c m}^{-\mathbf{3}}\right)$ & $\mathbf{t}(\mathbf{p s})$ & $\mathbf{S}\left(\mathbf{c m}^{-\mathbf{3}}\right)$ & $\mathbf{t}(\mathbf{p s})$ & $\mathbf{S}\left(\mathbf{c m}^{-3}\right)$ & $\mathbf{t}(\mathbf{p s})$ \\
\hline $1.11 \times 10^{12}$ & 1.8272 & $8.37 \times 10^{11}$ & 2.0233 & $5.09 \times 10^{11}$ & 2.3189 \\
$1.67 \times 10^{11}$ & 3.0272 & $4.91 \times 10^{11}$ & 2.4233 & $2.23 \times 10^{11}$ & 3.1189 \\
$7.6 \times 10^{10}$ & 3.5272 & $1.79 \times 10^{11}$ & 3.5233 & $1.52 \times 10^{11}$ & 3.8595 \\
\hline
\end{tabular}

Now, we generalize rate equation for spin-dependent electron $\left(n_{ \pm}\right)$and photon $\left(S^{\mp}\right)$ densities related to QW spin-VCSEL. Thus, mathematical forms of these rate equations are $[21,22]$

$$
\begin{gathered}
\frac{d n_{ \pm}}{d t}=J_{ \pm}-\left(\frac{g_{0}\left(n_{ \pm}+p_{ \pm}-n_{\text {tran }}\right)\left(S^{\mp}\right)}{(1+\varepsilon S)}\right)-\frac{n_{ \pm}-n_{\mp}}{\tau^{n}{ }_{s}}-R^{ \pm}{ }_{s p} \\
\frac{d S^{ \pm}}{d t}=\Gamma\left(\frac{g_{0}\left(n_{\mp}+p_{\mp}-n_{\text {tran }}\right)\left(S^{ \pm}\right)}{(1+\varepsilon S)}\right)-\Gamma \beta R^{\mp}{ }_{s p}(n)-\frac{S^{ \pm}}{\tau_{p h}}
\end{gathered}
$$

Where $R_{s p}^{ \pm}$is spin-dependent spontaneous recombination function which has $\mathrm{LR}$ and $\mathrm{QR}$ form of spontaneous recombination with mathematical forms as $R_{s p}{ }^{ \pm}=n_{ \pm} / \tau_{r}$ and $R_{s p}{ }^{ \pm}=B n_{ \pm} p_{ \pm}=B n_{ \pm} n / 2 \cdot \frac{n_{ \pm}-n_{\mp}}{\tau^{n}{ }_{s}}$ and $S$

terms represent the electron spin relaxation and sum of photon densities with positive and negative helicity. Note that due to charge neutrality, we could decouple the rate equations for spin-dependent electrons from those for holes. Fig. 3 shows time dependency of spin-up electron densities by considering LR spontaneous recombination.

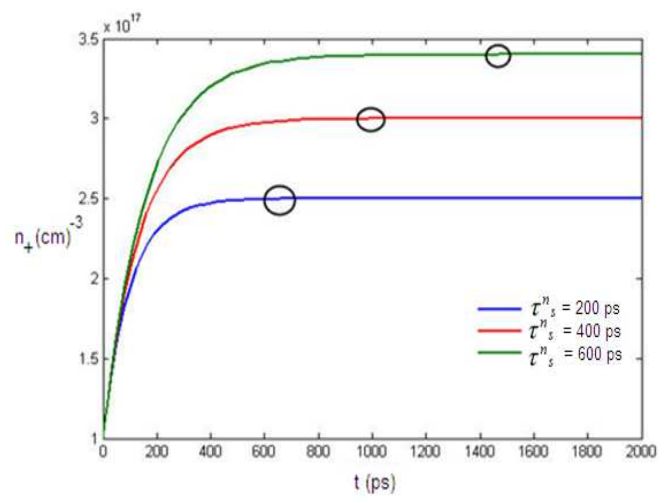

Fig 3. Time dependency of spin-up electron densities in a QW SSPL.

This figure implies that by rise of electron spin relaxation time, we observe increase of spin-up electron density at constant spin polarized electron injection $10^{15}$ per every second by considering LR spontaneous recombination As shown in Fig. 3, from points $\left(660.3900,2.50 \times 10^{17}\right)$ and $\left(986.3460,2.99 \times 10^{17}\right)$ per $\tau_{s}^{n}=200 p s$ and $\tau_{s}^{n}=400 p s$ which are characterized with black circles, spin-up electron densities have constant trend and saturation reaches. Table 3 illustrates obtained values of spin-up electron density for two various electron spin relaxation times at various times. 
Table 3. Obtained values of photon densities for two electron spin relaxation times at various times

\begin{tabular}{cccc}
\multicolumn{2}{c}{$\boldsymbol{\tau}_{\mathbf{s}}^{\mathbf{n}}=\mathbf{2 0 0} \mathbf{~ p s}$} & \multicolumn{2}{c}{$\boldsymbol{\tau}_{\mathbf{s}}^{\mathbf{n}}=\mathbf{4 0 0} \mathbf{~ p s}$} \\
$\mathbf{n}\left(\mathbf{c m}^{-3}\right)$ & $\mathbf{t}(\mathbf{p s})$ & $\mathbf{n}\left(\mathbf{c m}^{-\mathbf{3}}\right)$ & $\mathbf{t}(\mathbf{p s})$ \\
\hline $2.33 \times 10^{17}$ & 214.8174 & $2.61 \times 10^{17}$ & 219.4527 \\
$2.47 \times 10^{17}$ & 418.7178 & $2.92 \times 10^{17}$ & 422.7011 \\
$2.50 \times 10^{17}$ & 660.3900 & $2.99 \times 10^{17}$ & 986.3460 \\
\hline
\end{tabular}

Fig. 4 demonstrates time dependency of negative helicity photon densities by considering LR spontaneous recombination.

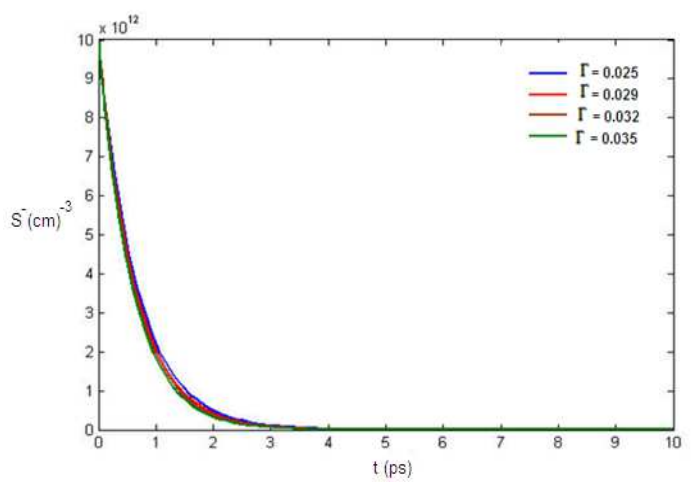

Fig 4. Time dependency of negative helicity photon densities for a $Q W$ SSPL.

According to figure 4, we find out decrease of photon densities at constant spin polarized electron injection $10^{15}$ per every second by considering LR spontaneous recombination. As shown in Fig. 4, we note that by increase of optical confinement factor, drop of negative helicity photon densities slower. Table 4 illustrates obtained values of negative helicity photon density for three various optical confinement factors at various times.

Table 4. Obtained values of negative helicity photon density for three various optical confinement factors at various times

\begin{tabular}{cccccc}
\hline \multicolumn{2}{c}{$\Gamma=0.029$} & \multicolumn{2}{c}{$\Gamma=\mathbf{0 . 0 3 2}$} & \multicolumn{2}{c}{$\Gamma=0.035$} \\
$\mathbf{S}^{-}\left(\mathbf{c m}^{-\mathbf{3}}\right)$ & $\mathbf{t}(\mathbf{p s})$ & $\mathbf{S}^{-}\left(\mathbf{c m}^{-3}\right)$ & $\mathbf{t}(\mathbf{p s})$ & $\mathbf{S}^{-}\left(\mathbf{c m}^{-3}\right)$ & $\mathbf{t}(\mathbf{p s})$ \\
\hline $1.06 \times 10^{11}$ & 2.8861 & $8.70 \times 10^{10}$ & 2.9005 & $7.17 \times 10^{10}$ & 2.9138 \\
$2.55 \times 10^{9}$ & 5.5192 & $2.31 \times 10^{9}$ & 5.4417 & $1.91 \times 10^{9}$ & 5.4959 \\
$9.27 \times 10^{8}$ & 8.7748 & $9.80 \times 10^{8}$ & 8.9433 & $1.03 \times 10^{9}$ & 8.8841 \\
\hline
\end{tabular}

\section{NSFI Width}

Creation of NSFI is one of important consequences of spin polarized injection. Width of this interval can be obtained analytically from rate equations (3) and (4) which can be presented for LR and QR spontaneous recombination as[21, 22]

$$
d=3\left|P_{J}\right| /\left(2-\left|P_{J}\right|-\left|P_{J}\right|^{2}\right)
$$

$$
d=\left|P_{J}\right|\left(8+\left|P_{J}\right|\right) /\left(4-3\left|P_{J}\right|^{2}-\left|P_{J}\right|^{3}\right)
$$

Where $\left|P_{J}\right|$ is polarization of injected electron current. Fig. 5 shows variations of NSFI versus polarization of injected electron current for two types of spontaneous recombination.

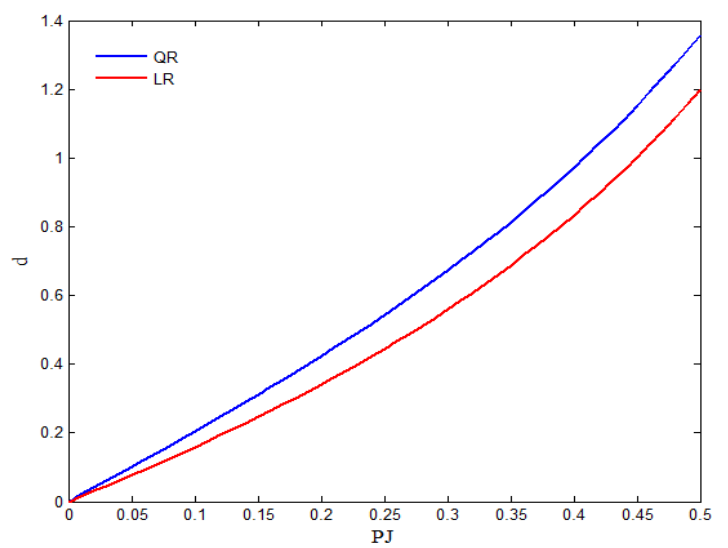

Fig 5. Variations of NSFI versus polarization of injected electron current for two types of spontaneous recombination.

From this above Fig, NSFI increases by rising of injected electron current polarization for two types of spontaneous recombination. Also, we found that this interval for LR spontaneous recombination is always smaller than those of QR. Table 5 is illustrated obtained values for NSFI related to two types of spontaneous recombination per three various polarization of injected electron current.

Table 5. Obtained values for NSFI per three various polarization of injected electron current related to two types of spontaneous recombination.

\begin{tabular}{cccccc}
\hline \multicolumn{2}{c}{$\left|P_{J}\right|=0.1$} & \multicolumn{2}{c}{$\left|P_{J}\right|=0.3$} & \multicolumn{2}{c}{$\left|P_{J}\right|=0.5$} \\
$\mathbf{d}(\mathbf{L R})$ & $\mathbf{d}(\mathbf{Q R})$ & $\mathbf{d}(\mathbf{L R})$ & $\mathbf{d}(\mathbf{Q R})$ & $\mathbf{d}(\mathbf{L R})$ & $\mathbf{d}(\mathbf{Q R})$ \\
\hline 0.16 & 0.2 & 0.56 & 0.67 & 1.2 & 1.36 \\
\hline
\end{tabular}

According to Table 5 and obtained values, NSFI maximum for LR and QR spontaneous recombination is 1.2 and 1.36 , respectively.

\section{TCDR}

One of spin polarized injection benefits is TCDR which results from creation of NSFI[23-25]. In this interval, only charge carrier with majority spin species contribute at lasing process. TCDR can be obtained analytically from rate equations (3) and (4) which can be presented for LR and QR spontaneous recombination as[21, 22]

$$
\begin{gathered}
r=\left|P_{J}\right| /\left(2+\left|P_{J}\right|+4 w\right) \\
r=1-\frac{\left[1-2 w+\sqrt{(1+2 w)^{2}+4\left|P_{J}\right| w}\right]^{2}}{\left(2+\left|P_{J}\right|\right)^{2}}
\end{gathered}
$$

Where $w$ is normalized spin relaxation rate which is 
defined as result of divide recombination time on electron spin relaxation time. Figs. 6 and 7 show variations of TCDR versus simultaneously polarization of injected electron current and normalized spin relaxation rate or two types of spontaneous recombination.

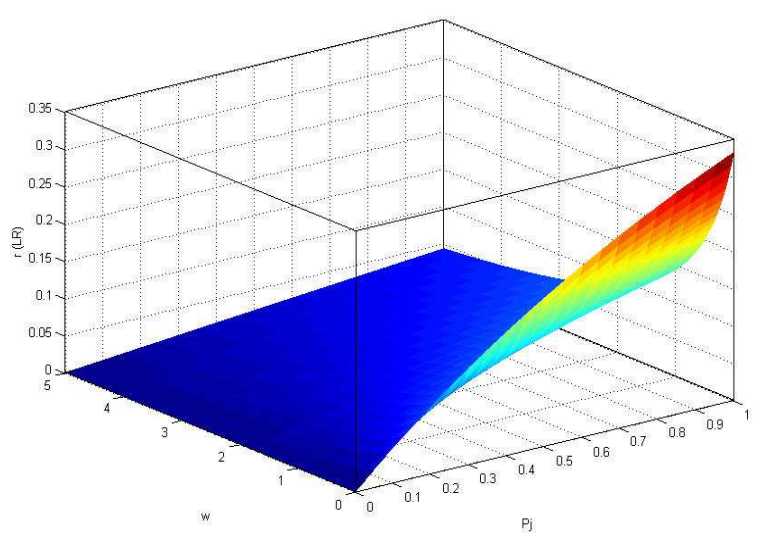

Fig 6. Variations of TCDR versus of polarization of injected electron current and normalized spin relaxation rate for $L R$ spontaneous recombination.

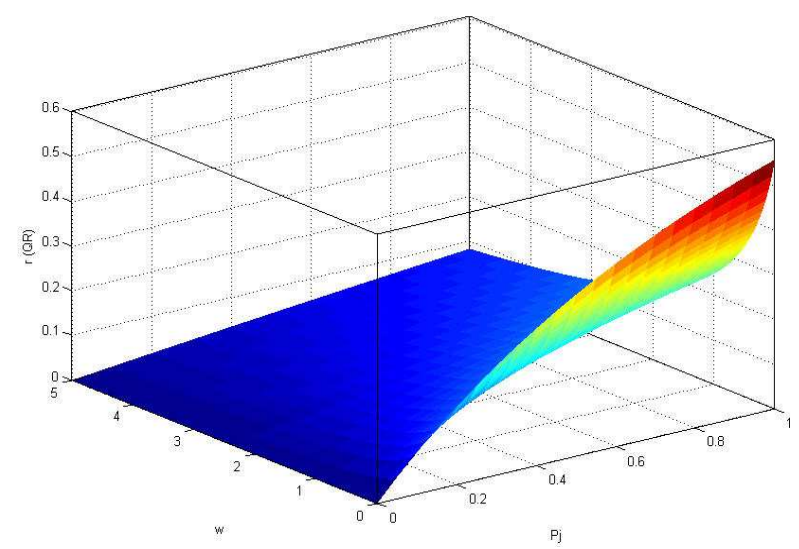

Fig 7. Variations of TCDR versus of polarization of injected electron current and normalized spin relaxation rate for $Q R$ spontaneous recombination.

Figs. 9 and 10 present variations of TCDR versus polarization of injected electron current for two types of spontaneous recombination.

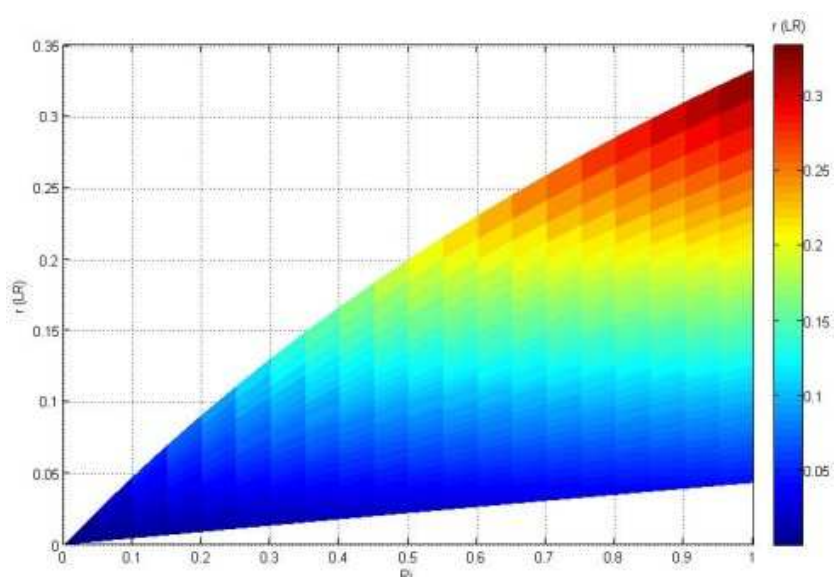

Fig 8. Variations of TCDR versus polarization of injected electron current for LR spontaneous recombination.

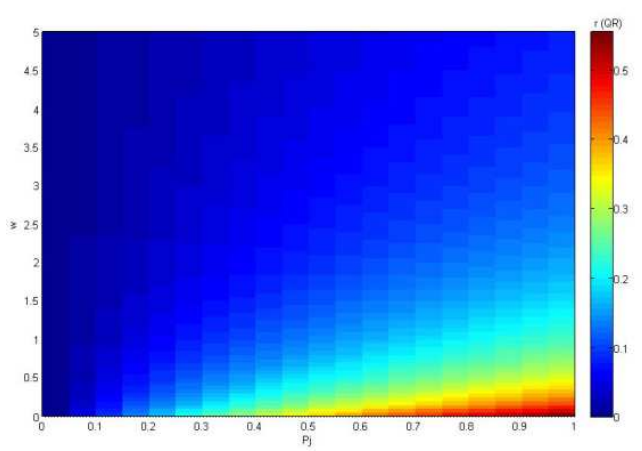

Fig 9. Variations of TCDR versus polarization of injected electron current for $Q R$ spontaneous recombination.

In Figs.6-9, dark red and blue areas demonstrate highest and lowest TCDR, respectively. According to these Figs, we find out that it increases with simultaneously normalized spin relaxation rate reduction and increase of polarization of injected electron current. Such a reduction decreases the power consumption of lasers and enhances laser dynamic performance. This advantage is obtained by using electrical spin injection in QW SSPL. Increase of electrical spin injection leads to raise polarization of injected electron current and laser bandwidth. Note that TCDR is always smaller than those of QR. Figs. 10 and 11 showvariations of TCDR versus normalized spin relaxation rate per three various polarizations of injected electron currents related to two types of spontaneous recombination.

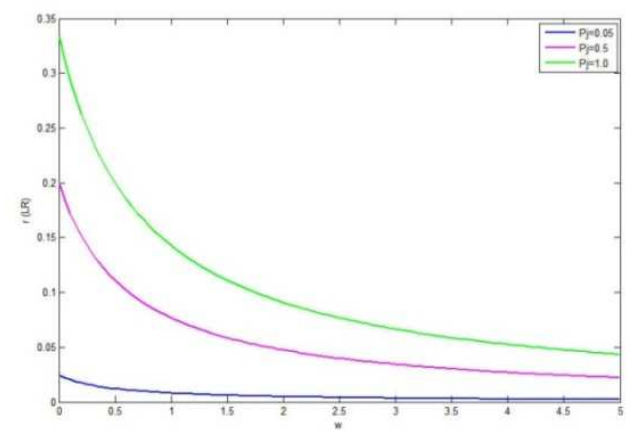

Fig 10. Variations of TCDR versus normalized spin relaxation rate per three various polarizations of injected electron currents for LR spontaneous recombination.

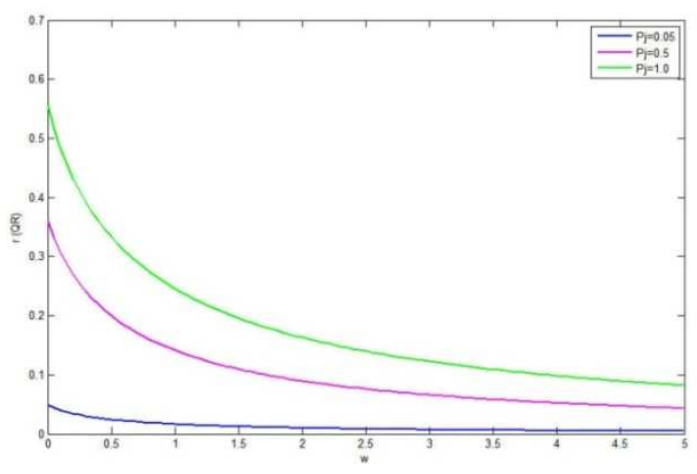

Fig 11. Variations of TCDR versus normalized spin relaxation rate per three various polarizations of injected electron currents for $Q R$ spontaneous recombination 
According to Figs. 10 and 11, per a specific injected electron current polarization, TCDR grows up by normalized spin relaxation rate reduction. Also, such a reduction increases via rise of injected electron current polarization. Table 6 is illustrated obtained values for TCDR related to two types of spontaneous recombination.

Table 6. Obtained values for TCDR per two various injected electron current polarization related to two types of spontaneous recombination.

\begin{tabular}{|c|c|c|c|c|c|c|c|}
\hline \multicolumn{4}{|c|}{$\left|P_{J}\right|=0.1$} & \multicolumn{4}{|c|}{$\left|P_{J}\right|=0.5$} \\
\hline \multicolumn{2}{|c|}{$W=1$} & \multicolumn{2}{|c|}{$W=5$} & \multicolumn{2}{|c|}{$\mathrm{W}=1$} & \multicolumn{2}{|c|}{$W=5$} \\
\hline $\begin{array}{c}\mathbf{r} \\
(\mathbf{L R})\end{array}$ & $\begin{array}{c}\mathbf{r} \\
(\mathbf{Q R})\end{array}$ & $\begin{array}{c}\mathbf{r} \\
(\mathbf{L R})\end{array}$ & $\begin{array}{c}\mathbf{r} \\
(\mathbf{Q R})\end{array}$ & $\begin{array}{c}\mathbf{r} \\
(\mathbf{L R})\end{array}$ & $\begin{array}{c}\mathbf{r} \\
(\mathbf{Q R})\end{array}$ & $\begin{array}{c}\mathbf{r} \\
(\mathbf{L R})\end{array}$ & $\begin{array}{c}\mathbf{r} \\
(\mathbf{Q R})\end{array}$ \\
\hline 0.016 & 0.04 & 0.004 & 0.02 & 0.07 & 0.15 & 0.022 & 0.31 \\
\hline
\end{tabular}

Based on Table 6 and obtained values, TCDR maximum for LR and QR spontaneous recombination is 0.07 and 0.31 , respectively.

\section{Optical Gain}

The optical gain describes coupling of the carriers and light, which gives rise to stimulated emission [26, 27]. According to importance of this quantity, we intend to investigate variation of spin-dependent optical gain versus spin-up electron density and gain compression factors. Spin-dependent gain term can be written as [21, 22]

$$
g_{ \pm}=g_{0} \frac{\left(n_{ \pm}+p_{ \pm}-n_{t r a n}\right)}{(1+\varepsilon S)}
$$

Where $S$ is total photon density with left and right circular polarization.Fig.12 shows variation of spin-up optical gain versus spin-up electron density and gain compression factor. Spin-up gain term increases by gain compression factor reduction but in the case spin-up electron density, by its rising variation of spin-up optical gain have constant trend.

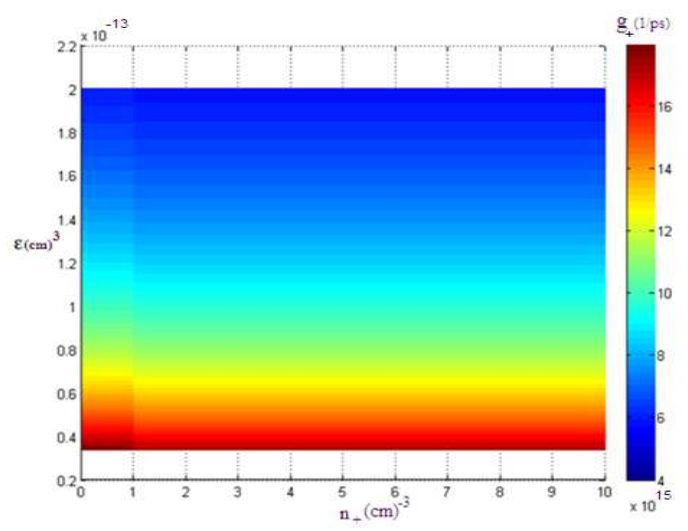

Fig 12. Variation of Spin-up optical gain versus spin-up electron density and gain compression factor

Increase of spin-up optical gain ensures efficiency of laser. Table 7 demonstrates obtained values for spin-up optical gain for two various spin-up electron densities.
Table 7. Obtained values of spin-up optical gain for two various spin-up electron densities

\begin{tabular}{cccc}
\multicolumn{2}{c}{$\mathbf{n}_{+}=\mathbf{1 0}^{\mathbf{1 6}}$} & \multicolumn{2}{c}{$\mathbf{n}_{+}=\mathbf{1 0}^{\mathbf{1 7}}$} \\
$\boldsymbol{\varepsilon}$ & $\mathbf{g}_{+}$ & $\boldsymbol{\varepsilon}$ & $\mathbf{g}_{+}$ \\
\hline $3.35 \times 10^{-14}$ & 17.36 & $3.35 \times 10^{-14}$ & 11.98 \\
$1.14 \times 10^{-13}$ & 8.82 & $1.14 \times 10^{-13}$ & 6.09 \\
$2 \times 10^{-13}$ & 5.8 & $2 \times 10^{-13}$ & 4 \\
\hline
\end{tabular}

Based on Table 7 and obtained values, spin-up optical gain maximum is 17.36 , respectively.

\section{Conclusion and Discussion}

According to above discussion, it appearances intensive dependency of QW SSPL operations on spin injection and longer spin relaxation time. We demonstrate for the first time, simultaneously effect of normalized spin relaxation rate and injected current polarization on TCDR related to two form of spontaneous recombination. According to our result, TCDR increases by simultaneously normalized spin relaxation rate reduction and increasing of injected current polarization. Maximum obtained TCDR values for LR and QR spontaneous recombination is 0.07 and 0.31 . In addition to, we investigate injected current polarization effect on NSFI related to two form of spontaneous recombination. We found that NSFI increases by growing up injected current polarization. Maximum obtained NSFI values for LR and QR spontaneous recombination 1.2 and 1.36.Spin-up optical gain term increases by gain compression factor reduction. Maximum obtained Spin-up optical gain 17.36.

\section{References}

[1] S. L. Chuang, Physics of Optoelectronic Devices, 2nded. Wiley, New York, (2009).

[2] M. A. Parker, Physics of Optoelectronics, CRC, New York, (2004).

[3] L. A. Coldren and S. W. Corzine, Diode Lasers and Photonic Integrated Circuits, (Wiley, New York, (1995).

[4] W. W. Chow and S. W. Koch, Semiconductor Laser Fundamentals: Physics of the Gain Materials Springer, NewYork, (1999).

[5] H. Haken, Light, Vol. 2 Laser Light Dynamics NorthHolland, New York, 1985.

[6] Z. I. Alferov, Rev. Mod. Phys. 73, 767 (2001).

[7] V. M. Ustinov, A. E. Zhukov, A. Yu. Egorov, and N. A.Maleev,Quantum Dot Lasers(Oxford University Press, New York, (2003).

[8] D. Bimberg, M. Grundmann, and N. N. Ledentsov, Quanutm Dot Heterostructures, Wiley, New York, (1999).

[9] A. Das, J. Heo, M. Jankowski, W. Guo, L. Zhang, H. Deng, and P. Bhattacharya, Phys. Rev. Lett.107, 066405 (2011). 
[10] J. Rudolph, D. H“agele, H. M. Gibbs, G. Khitrova, and M.Oestreich, Appl. Phys. Lett.82, 4516 (2003).

[11] J. Rudolph, S. D“ohrmann, D. H“agele, M. Oestreich, and W. Stolz, Appl. Phys. Lett.87, 241117 (2005).

[12] M. Holub, J. Shin, and P. Bhattacharya, Phys. Rev. Lett. 98, 146603 (2007).

[13] S. Iba, S. Koh, K. Ikeda, and H. Kawaguchi, Room temperature circularly polarized lasing in an optically spin injected vertical-cavity surface-emitting laser with (110) GaAs quantum wells,Appl. Phys. Lett. 98, 081113 (2011).

[14] M. Oestreich, J. Rudolph, R. Winkler, and D. Hagele, Design considerations for semiconductor spin lasers, SuperlatticesMicrostruct. 37, 306-312 (2005).

[15] C. Gothgen, R. Oszwałdowski, A. Petrou, and I. Žutić, Analytical model of spin-polarized semiconductor lasers, Appl. Phys. Lett. 93, 042513 (2008).

[16] J. Lee, W. Falls, R. Oszwałdowski, and I. Žutić, Spin modulation in semiconductor lasers, Appl. Phys. Lett. 97, 041116 (2010).

[17] G. Schmidt, D. Ferrand, L. W. Molenkamp, A. T. Filip, and B. J. van Wees;"Fundamental obstacle for electrical spin injection from a ferromagnetic metal into a diffusive semiconductor”; Phys. Rev. B62, R4790 (2000).

[18] E. I. Rashba;"Theory of electrical spin injection: Tunnel contacts as a solution of the conductivity mismatch problem"; Phys. Rev. B62, R16267 (2000).
[19] A. Fert and H. Jaffres;"Conditions for efficient spin injection from a ferromagnetic metal into a semiconductor"; Phys. Rev. B64, 184420 (2001).

[20] R. A. de Groot, F. M. Mueller, P. G. v. Engen, and K. H. J. Buschow; "New class of materials: Half-metallic ferromagnets";Phys. Rev. Lett. 50, 2024 (1983).

[21] J. Lee, R. Oszwałdowski, C. Gøthgen, and I. Žutić; "Mapping between quantum dot and quantum well lasers: From conventional to spin lasers"; Phys. Rev. B 85, 045314 (2012).

[22] EEY. Tsymbal and I. Žutić; Handbook of Spin Transport and Magnetism, CRCPress. 731-745 (2012).

[23] J. Rudolph, D. Hägele, H. M. Gibbs, G. Khitrova, and . M. Oestreich, Laser threshold reduction in a spintronic device, Appl. Phys. Lett. 82, 4516-4518 (2003).

[24] J. Rudolph, S. Dohrmann, D. Hagele, M. Oestreich, and W. Stolz, Room-temperature threshold reduction in verticalcavity surface-emitting lasers by injection of spin-polarized carriers, Appl. Phys. Lett. 87, 241117 (2005).

[25] M. Holub, J. Shin, and P. Bhattacharya, Electrical spin injection and threshold reduction in a semiconductor laser, Phys. Rev. Lett. 98, 146603 (2007).

[26] D. Basu, D. Saha, and P. Bhattacharya, Optical polarization modulation and gain anisotropy in an electrically injected spin laser, Phys. Rev. Lett. 102, 093904 (2009).

[27] R. Oszwałdowski, C. Gøthgen, and I. Žutić, Theory of quantum dot spin-lasers, Phys. Rev. B82, 085316 (2010). 